\title{
Assessment of Metabolic Phenotypes in Patients with Non-ischemic Dilated Cardiomyopathy Undergoing Cardiac Resynchronization Therapy
}

\author{
Sebastian Obrzut • Jay Tiongson • Neema Jamshidi • \\ Huy Minh Phan • Carl Hoh • \\ Ulrika Birgersdotter-Green
}

Received: 27 May 2010 / Accepted: 1 September 2010/Published online: 15 September 2010

(C) The Author(s) 2010. This article is published with open access at Springerlink.com

\begin{abstract}
Studies of myocardial metabolism have reported that contractile performance at a given myocardial oxygen consumption (MVO2) can be lower when the heart is oxidizing fatty acids rather than glucose or lactate. The objective of this study is to assess the prognostic value of myocardial metabolic phenotypes in identifying nonresponders among non-ischemic dilated cardiomyopathy (NIDCM) patients undergoing cardiac resynchronization therapy (CRT). Arterial and coronary sinus plasma concentrations of oxygen, glucose, lactate, pyruvate, free fatty acids (FFA), and 22 amino acids were obtained from 19 male and 2 female patients (mean age $56 \pm 16$ ) with NIDCM undergoing CRT. Metabolite fluxes/MVO2 and extraction fractions were calculated. Flux balance analysis (FBA) was performed with MetaFluxNet 1.8 on a metabolic network of the cardiac mitochondria (189 reactions, 230 metabolites) reconstructed from mitochondrial proteomic data $(615$ proteins) from human heart tissue. Non-responders based on left ventricular ejection fraction (LVEF) demonstrated a greater mean FFA extraction fraction $(35 \% \pm 17 \%)$ than responders $[18 \pm 10 \%, p=0.0098$, area under the estimated
\end{abstract}

\footnotetext{
S. Obrzut $\cdot$ N. Jamshidi $\cdot$ C. Hoh

Division of Nuclear Medicine,

University of California San Diego,

La Jolla, CA, USA

J. Tiongson $\cdot$ H. M. Phan • U. Birgersdotter-Green

Department of Cardiology, University of California San Diego,

La Jolla, CA, USA

S. Obrzut $(\bowtie)$

UCSD Medical Center, Hillcrest, Division of Nuclear Medicine, 200 West Arbor Drive,

San Diego, CA 92103-8758, USA

e-mail: sobrzut@ucsd.edu
}

ROC curve (AUC) was 0.8238, S.E. 0.1115]. Calculated adenosine triphosphate (ATP)/MVO2 using FBA correlated with change in New York Heart Association (NYHA) class $($ rho $=0.63, p=0.0298 ; \mathrm{AUC}=0.8381$, S.E. 0.1316). Nonresponders based on both LVEF and NYHA demonstrated a greater mean FFA uptake/MVO2 $(0.115 \pm 0.112)$ than responders $(0.034 \pm 0.030, p=0.0171 ; \mathrm{AUC}=0.8593$, S.E. 0.0965). Myocardial FFA flux and calculated maximal ATP synthesis flux using FBA may be helpful as biomarkers in identifying non-responders among NIDCM patients undergoing CRT.

Keywords Metabolism · Fatty acids · Cardiomyopathy

\section{Introduction}

Cardiac resynchronization therapy (CRT) has been shown to reduce morbidity and mortality as well as improve left ventricular (LV) function and reverse remodeling in patients with advanced heart failure [1-4]. However, not all patients respond to CRT and the overall nonresponse rate is estimated to be approximately $30 \%$ [5]. Two multicenter trials were unable to demonstrate a prognostic value of mechanical markers of LV function in patients undergoing CRT $[6,7]$. Cardiac imaging methods are still in an emerging state for prediction of CRT outcome [8]. Therefore, identification of biomarkers which can predict CRT response in heart failure patients would be of great clinical interest.

Heart failure patients have been shown to exhibit alterations of myocardial metabolism, which may contribute to the contractile dysfunction and to the progression of left ventricular remodeling [9]. However, studies of 
alterations in myocardial energy metabolism in heart failure have been contradictory [10-16]. In addition, the role of many important metabolites in heart failure, including lactate and glutamate, has not been well described, limiting the application of emerging constraint based systems biology computational methods, such as flux balance analysis (FBA), to more completely analyze the myocardial substrate metabolism [17]. It has been reported that contractile performance of the heart can vary depending on the metabolic phenotype. For instance, at a given myocardial oxygen consumption (MVO2), contractile performance can be lower when the heart is oxidizing fatty acids and greater when the heart is oxidizing other substrates such as glucose and lactate [9, 18-21]. We hypothesized that myocardial metabolic phenotypes may demonstrate prognostic value in heart failure patients undergoing CRT. In this study we employed FBA, a system-based approach, to evaluate substrate metabolism in patients with non-ischemic dilated cardiomyopathy (NIDCM) undergoing biventricular pacemaker-implantable cardioverter defibrillator (BiV ICD) implantation.

\section{Methods}

\section{Study Population}

In this prospective study, 21 NIDCM patients (19 male, 2 female; mean age $56 \pm 16$ years) receiving optimal, individualized therapy for heart failure underwent implantation of a BiV ICD based on standard indications for CRT. All patients underwent a complete cardiac evaluation, including a history, physical exam, echocardiogram and coronary angiography. Inclusion criteria for patients with NIDCM were: (1) diagnosis of cardiomyopathy within 12 months of enrollment; (2) no evidence of coronary artery disease (CAD) as assessed by coronary angiography; (3) global moderate-tosevere left ventricular dysfunction and dyssynchrony. All patients were required to be clinically stable for at least one month before completing the study protocol and fast for more than $12 \mathrm{~h}$ prior to undergoing BiV ICD implantation. Written informed consent, approved by the Human Research Protection Program, was obtained from all study subjects.

\section{Assessment of Myocardial Metabolism}

Blood was drawn from the coronary sinus and then immediately form the radial artery from all patients at the time of BiV ICD implantation. Arterial and venous concentrations of oxygen, glucose, lactate and pyruvate were evaluated following blood drawing by the laboratory at our institution. Furthermore, blood samples were submitted to ARUP Laboratories (Salt Lake City, UT) for evaluation of arterial and venous plasma concentrations of free fatty acids (FFA), glutamate, aspartate, alanine, glutamine, arginine, citrulline, cysteine, glycine, histidine, hydroxyproline, isoleucine, leucine, lysine, methionine, ornithine, phenylalanine, proline, serine, taurine, threonine, tyrosine, and valine. Arterial plasma insulin levels were also measured for each subject.

The myocardial flux of a substrate can be expressed as the product of the substrate arterial-coronary sinus concentration difference and myocardial blood flow [22]. This metabolic flux can be corrected for MVO2 and expressed as the ratio of arterial-coronary sinus concentration difference for each metabolite to the arterial-coronary sinus concentration difference of oxygen, eliminating the need to measure myocardial blood flow, as previously described by Vanky et al. [22]. Therefore, myocardial metabolic fluxes of all measured substrates, corrected for MVO2, were calculated. In addition, myocardial extraction fraction (ExtFx) for each metabolite was calculated as the ratio of the metabolite arterial-coronary sinus concentration difference and the metabolite arterial concentration.

\section{Flux Balance Analysis}

FBA was performed on a metabolic network of the human cardiac mitochondria (189 reactions, 230 metabolites), which was previously reconstructed from published mitochondrial proteomic data (615 proteins) from human heart tissue [23]. The metabolic fluxes acquired from patients with NIDCM and corrected for MVO2 were used as constraints for FBA. Optimal steady-state flux distributions of the metabolic network were calculated with MetaFluxNet 1.8 [24]. An objective function maximizing ATP production was chosen for the calculations and maximal ATP synthesis flux, corrected for MVO2, was obtained.

\section{Clinical Response Criteria}

New York Heart Association (NYHA) class was assessed prior to BiV ICD (Pre-NYHA) implantation and $8.5 \pm$ 5.4 months following implantation (Post-NYHA). Patients were classified as responders based on NYHA class (NYHA-R) when the class decreased following implantation and as non-responders (NYHA-NR) if the class remained unchanged or increased.

Furthermore, echocardiograms were obtained prior to BiV ICD implantation and $6.5 \pm 4.5$ months after implantation. Left ventricular ejection fraction (Pre-LVEF), left ventricular end diastolic diameter (Pre-LVEDDM), and left atrial diameter (Pre-LAD) were assessed on the echocardiograms prior to implantation and after implantation (PostLVEF, Post-LVEDDM, Post-LAD). Patients were classified as responders based on LVEF (LVEF-R) when the LVEF 
increased by more than $5 \%$ and as non-responders (LVEF$\mathrm{NR}$ ) if these response criteria were not satisfied.

In addition, patients who responded based on both the NYHA class and LVEF response criteria were labeled as complete responders (C-R). All patients who responded based on either NHYA class or LVEF criteria were labeled as all-responders (All-R). Finally, patients who did not respond based on neither the NYHA class nor the LVEF criteria were labeled as complete non-responders (C-NR). Clinicians who performed echocardiograms and assessed the NYHA class in all patients were blinded to the metabolic data obtained at the time of implantation.

\section{Statistical Analysis}

Sign test was used to assess uptake or secretion of each metabolite. Student's $t$ test was used to compare mean LVEF, LVEDDM, and LAD, while Mann-Whitney $U$ test was used to compare NYHA class in patients before and after BiV ICD implantation [25]. Spearman correlations were evaluated between arterial insulin concentration, patient age, sex, and metabolite fluxes/MVO2 as well as metabolite extraction fractions. Nonparametric Spearman correlations were also used to evaluate metabolic data in NYHA-R and NYHA-NR patients [25, 26]. Student's $t$ test was used to compare metabolic data in LVEF-R and LVEFNR patients, as well as in C-NR, C-R, and All-R patients. Receiver-operating characteristic (ROC) curves were con- structed using the ROCKIT 0.9B software package for metabolic data which demonstrated statistical significance based on Student's $t$ test or nonparametric Spearman correlation [26].

\section{Results}

Demographics and Clinical Results

Patient demographics, clinical history, and type of nonischemic heart failure are shown in Table 1, while Table 2 shows hemodynamic parameters, LVEF, NYHA class, LVEDDM, and LAD in non-responders and responders to CRT. Mean Pre-LVEF was $23 \% \pm 8 \%$, which increased to $30 \% \pm 13 \%$ status post-placement of $\mathrm{BiV}$ ICD ( $p=$ 0.043). Furthermore, the NYHA class decreased status post-BiV ICD implantation $(p=0.0049)$. Mean plasma glucose was $116 \pm 48(\mathrm{mg} / \mathrm{dl})$. LVEF-NR patients were younger than LVEF-R patients $(p=0.02)$. C-NR patients demonstrated lower systolic blood pressure than All-R patients $(p=0.04)$.

\section{Metabolic Results}

There was net myocardial uptake of oxygen, glucose, FFA, lactate, glutamate, aspartate $(p<0.05)$, and net myocardial secretion of alanine $(p<0.05)$. There was no statistically
Table 1 Patient demographics (mean $\pm \mathrm{SD}$ ), clinical history, and type of non-ischemic heart failure in non-responders and responders to CRT

Statistically significant differences between means $(p<0.05)$ are marked with *

\begin{tabular}{|c|c|c|c|c|c|}
\hline & \multicolumn{2}{|c|}{ Non-responders } & \multicolumn{3}{|l|}{ Responders } \\
\hline & LVEF-NR & C-NR & LVEF-R & $\mathrm{C}-\mathrm{R}$ & All-R \\
\hline Age & $46 \pm 11 *$ & $45 \pm 9$ & $63 \pm 17^{*}$ & $62 \pm 22$ & $57 \pm 17$ \\
\hline Body weight (kg) & $85.9 \pm 18.8$ & $75.9 \pm 12.9$ & $84.0 \pm 18.0$ & $82.0 \pm 10.7$ & $87.6 \pm 18.6$ \\
\hline Height $(\mathrm{cm})$ & $135.1 \pm 75.9$ & $128.6 \pm 84.8$ & $174.9 \pm 12.3$ & $174.0 \pm 5.9$ & $161.6 \pm 49.2$ \\
\hline Body mass index $\left(\mathrm{kg} / \mathrm{m}^{2}\right)$ & $29.1 \pm 7.8$ & $26.1 \pm 5.8$ & $26.6 \pm 3.3$ & $26.5 \pm 4.4$ & $28.5 \pm 6.3$ \\
\hline Current smoker & $2 / 9$ & $1 / 4$ & $1 / 11$ & $0 / 5$ & $2 / 15$ \\
\hline Hypercholesterolemia & $4 / 9$ & $1 / 4$ & $7 / 11$ & $3 / 5$ & $9 / 15$ \\
\hline ACE inhibitors & $9 / 9$ & $4 / 4$ & $7 / 11$ & $3 / 5$ & $11 / 15$ \\
\hline Beta-blockers & $8 / 9$ & $3 / 4$ & $11 / 11$ & $5 / 5$ & $15 / 15$ \\
\hline Diuretics & $8 / 9$ & $3 / 4$ & $9 / 11$ & $4 / 5$ & $13 / 15$ \\
\hline Digoxin & $6 / 9$ & $4 / 4$ & $5 / 11$ & $3 / 5$ & $7 / 15$ \\
\hline Angiotensin II blocker & $1 / 9$ & $1 / 4$ & $4 / 11$ & $2 / 5$ & $4 / 15$ \\
\hline Cholesterol lowering & $5 / 9$ & $1 / 4$ & $7 / 11$ & $3 / 5$ & $10 / 15$ \\
\hline \multicolumn{6}{|l|}{ NIDCM-type } \\
\hline Idiopathic & $2 / 9$ & $1 / 4$ & $8 / 11$ & $3 / 5$ & $8 / 15$ \\
\hline ETOH & $2 / 9$ & $2 / 4$ & $1 / 11$ & $1 / 5$ & $1 / 15$ \\
\hline Drug & $3 / 9$ & $1 / 4$ & $1 / 11$ & $1 / 5$ & $3 / 15$ \\
\hline Myocarditis & $1 / 9$ & $0 / 4$ & $0 / 11$ & $0 / 5$ & $1 / 15$ \\
\hline Peripartum & $1 / 9$ & $0 / 4$ & $0 / 11$ & $0 / 5$ & $1 / 15$ \\
\hline Familial dilated & $0 / 9$ & $0 / 4$ & $1 / 11$ & $0 / 5$ & $1 / 15$ \\
\hline
\end{tabular}


Table 2 Comparison of hemodynamic parameters, LVEF, NYHA class, LVEDDM, and $\mathrm{LAD}($ mean $\pm \mathrm{SD})$ in nonresponders and responders to CRT

Statistically significant differences between means $(p<0.05)$ are marked with *

\begin{tabular}{lcccccc}
\hline \multicolumn{3}{c}{ Non-responders } & \multicolumn{3}{l}{ Responders } \\
\cline { 2 - 3 } \cline { 6 - 7 } & LVEF-NR & C-NR & & LVEF-R & C-R & All-R \\
\hline HR (beats/min) & $79 \pm 13$ & $76 \pm 13$ & & $76 \pm 18$ & $76 \pm 18$ & $78 \pm 17$ \\
SBP (mm Hg) & $108 \pm 13$ & $98 \pm 11^{*}$ & & $118 \pm 18$ & $118 \pm 16$ & $117 \pm 16^{*}$ \\
DBP (mm Hg) & $66 \pm 11$ & $62 \pm 9$ & & $65 \pm 6$ & $63 \pm 7$ & $66 \pm 9$ \\
RPP (mm Hg×bt/min) & $8,491 \pm 1,941$ & $7,460 \pm 1,778$ & & $8,938 \pm 1,847$ & $8,996 \pm 2,050$ & $9,137 \pm 1,820$ \\
LV mass (g) & $388.8 \pm 68.7$ & $363.0 \pm 97.3$ & & $329.7 \pm 125.1$ & $321.8 \pm 73.4$ & $360.2 \pm 111.6$ \\
LVESD (cm) & $6.1 \pm 0.5$ & $6.0 \pm 0.3$ & & $6.3 \pm 1.3$ & $6.5 \pm 1.0$ & $6.3 \pm 1.1$ \\
Pre-EF (\%) & $25 \pm 6^{*}$ & $27 \pm 6$ & & $22 \pm 9 *$ & $18 \pm 11$ & $22 \pm 8$ \\
Post-EF (\%) & $24 \pm 9$ & $23 \pm 9$ & & $35 \pm 14$ & $31 \pm 15$ & $31 \pm 14$ \\
NYHA Pre & $3.1 \pm 0.6$ & $2.8 \pm 0.5$ & & $2.9 \pm 0.6$ & $3.2 \pm 0.4$ & $3.1 \pm 0.6$ \\
NYHA Post & $2.4 \pm 0.7$ & $3.0 \pm 0.8^{*}$ & & $2.4 \pm 0.5$ & $2.2 \pm 0.4$ & $2.3 \pm 0.5 *$ \\
Pre-LVEDDM (cm) & $7.0 \pm 0.6$ & $6.6 \pm 0.3$ & & $6.9 \pm 1.3$ & $7.3 \pm 0.9$ & $7.1 \pm 1.1$ \\
Post-LVEDDM (cm) & $7.2 \pm 0.9$ & $6.6 \pm 0.5$ & & $6.4 \pm 1.3$ & $6.5 \pm 1.2$ & $6.9 \pm 1.3$ \\
Pre-LAD (cm) & $5.3 \pm 0.6$ & $5.4 \pm 0.8$ & & $5.1 \pm 1.0$ & $5.6 \pm 0.8$ & $5.1 \pm 0.8$ \\
Post-LAD (cm) & $5.2 \pm 0.7$ & $5.3 \pm 0.9$ & & $5.0 \pm 0.9$ & $5.3 \pm 0.5$ & $5.0 \pm 0.8$ \\
\hline
\end{tabular}

significant evidence of myocardial uptake or secretion of pyruvate, glutamine, arginine, citrulline, cysteine, glycine, histidine, hydroxyproline, isoleucine, leucine, lysine, methionine, ornithine, phenylalanine, proline, serine, taurine, threonine, tyrosine, or valine. Table 3 shows atrial-venous concentration differences (AVdiff) and arterial-venous concentration differences normalized to ventricular mass (nVM) for all metabolites demonstrating significant myocardial uptake or secretion in non-responders and responders to CRT. LVEF-NR patients demonstrated greater FFA-
AVdiff and FFA-nVM than LVEF-R patients $(p=0.02$ and 0.04 , respectively). No significant correlation was found between arterial insulin concentration, blood glucose, and metabolite extraction fractions or metabolite fluxes/MVO2.

Identifications of Non-responders Based on LVEF

There were 9 patients (45\%) classified as LVEF-NR and 11 patients classified as LVEF-R. Follow-up Post-LVEF was not available in one patient. Extraction fractions for all

Table 3 Arterial-venous concentration differences (AVdiff) and arterial-venous concentration differences normalized to ventricular mass (nVM) for all metabolites demonstrating significant myocardial uptake or secretion in non-responders and responders $(\operatorname{mean} \pm \mathrm{SD})$

\begin{tabular}{|c|c|c|c|c|c|}
\hline & \multicolumn{2}{|l|}{ Non-responders } & \multicolumn{3}{|l|}{ Responders } \\
\hline & LVEF-NR & $\mathrm{C}-\mathrm{NR}$ & LVEF-R & $\mathrm{C}-\mathrm{R}$ & All-R \\
\hline Alanine-AVdiff $(\mu \mathrm{mol} / \mathrm{l})$ & $-21.2 \pm 28.2$ & $-23.5 \pm 30.4$ & $-19.4 \pm 33.0$ & $-33.6 \pm 26.0$ & $-17.5 \pm 31.1$ \\
\hline Aspartate-AVdiff $(\mu \mathrm{mol} / \mathrm{l})$ & $2.6 \pm 8.7$ & $-2.5 \pm 8.5$ & $0.7 \pm 2.1$ & $-0.2 \pm 0.4$ & $2.7 \pm 5.1$ \\
\hline FFA-AVdiff $(\mu \mathrm{mol} / \mathrm{l})$ & $315.6 \pm 232.3^{*}$ & $335.0 \pm 151.5$ & $123.6 \pm 86.5^{*}$ & $138.0 \pm 106.2$ & $185.3 \pm 194.8$ \\
\hline Glucose-AVdiff $(\mu \mathrm{mol} / \mathrm{l})$ & $238.3 \pm 481.1$ & $247.5 \pm 222.3$ & $315.0 \pm 1212.5$ & $286.0 \pm 284.2$ & $289.7 \pm 1083.3$ \\
\hline Glutamate-AVdiff $(\mu \mathrm{mol} / \mathrm{l})$ & $50.3 \pm 33.2$ & $33.8 \pm 43.6$ & $29.1 \pm 21.8$ & $27.4 \pm 27.9$ & $41.5 \pm 25.9$ \\
\hline Lactate-AVdiff $(\mu \mathrm{mol} / \mathrm{l})$ & $325.6 \pm 347.8$ & $50.0 \pm 92.6$ & $222.0 \pm 287.4$ & $31.1 \pm 79.8$ & $336.7 \pm 331.5$ \\
\hline O2-AVdiff $(\mu \mathrm{mol} / \mathrm{l})$ & $5,107 \pm 1,787$ & $4,126 \pm 2,184$ & $4,478 \pm 888$ & $4,256 \pm 895$ & $4,962 \pm 1,154$ \\
\hline Alanine-nVM $(\mu \mathrm{mol} / 1 / \mathrm{g})$ & $-0.063 \pm 0.080$ & $-0.079 \pm 0.095$ & $-0.049 \pm 0.136$ & $-0.134 \pm 0.119$ & $-0.042 \pm 0.116$ \\
\hline Aspartate-nVM $(\mu \mathrm{mol} / 1 / \mathrm{g})$ & $0.002 \pm 0.029$ & $-0.014 \pm 0.036$ & $0.001 \pm 0.006$ & $-0.001 \pm 0.002$ & $0.006 \pm 0.013$ \\
\hline FFA-nVM $(\mu \mathrm{mol} / \mathrm{l} / \mathrm{g})$ & $0.864 \pm 0.662 *$ & $1.017 \pm 0.642$ & $0.343 \pm 0.259^{*}$ & $0.369 \pm 0.298$ & $0.504 \pm 0.507$ \\
\hline Glucose-nVM $(\mu \mathrm{mol} / 1 / \mathrm{g})$ & $0.651 \pm 1.143$ & $0.735 \pm 0.621$ & $-0.431 \pm 6.348$ & $1.138 \pm 1.427$ & $-0.138 \pm 5.276$ \\
\hline Glutamate-nVM $(\mu \mathrm{mol} / \mathrm{l} / \mathrm{g})$ & $0.120 \pm 0.084$ & $0.074 \pm 0.105$ & $0.099 \pm 0.094$ & $0.120 \pm 0.135$ & $0.125 \pm 0.084$ \\
\hline Lactate-nVM $(\mu \mathrm{mol} / \mathrm{l} / \mathrm{g})$ & $0.787 \pm 0.879$ & $0.086 \pm 0.303$ & $0.944 \pm 1.423$ & $0.111 \pm 0.271$ & $1.143 \pm 1.243$ \\
\hline O2-nVM $(\mu \mathrm{mol} / 1 / \mathrm{g})$ & $13.54 \pm 4.93$ & $12.41 \pm 7.31$ & $14.22 \pm 4.04$ & $12.94 \pm 3.38$ & $14.40 \pm 3.58$ \\
\hline
\end{tabular}

Statistically significant differences between means $(p<0.05)$ are marked with * 
metabolites demonstrating significant myocardial uptake or secretion in responder and non-responder patients based on LVEF are compared in Table 4. LVEF-NR patients demonstrated a greater mean FFA-ExtFx $(35 \% \pm 17 \%)$ than LVEF-R patients (mean FFA-ExtFx $=18 \% \pm 10 \%, p=$ 0.0098), as shown on box plots in Fig. 1. The area under the estimated ROC curve (AUC) for FFA-ExtFx was 0.8238 (S.E. 0.1115) in the identification of LVEF-NR patients.

Identification of Non-responders Based on NYHA Class

There were 9 patients classified as NYHA-NR and 11 patients classified as NYHA-R. Follow-up post-NYHA class was not available in one patient. There was no significant correlation between the change in LVEF and the change in NYHA class in our patient population (Spearman's rho $=0.029, p=0.908$ ). Measured flux data for glucose/MVO2, lactate/MVO2, FFA/ $\mathrm{MVO} 2$, and glutamate/MVO2 were fully concordant with the FBA of the human cardiac mitochondria metabolic network in 12 patients. Therefore, for these patients, the flux data could be entered into MetaFluxNet 1.8 as exact constraints rather than maximal/minimal flux bounds. Maximal ATP synthesis flux, corrected for MVO2 (ATP/MVO2), was calculated. For the remaining patients, measured flux data were concordant with the FBA of the human cardiac mitochondria metabolic network only if entered into MetaFluxNet 1.8 as maximal/minimal flux bounds and were not used in subsequent analyses. Calculated ATP/MVO2 using FBA correlated with change in NYHA class (Spearman's rho $=0.63, p=0.0298$ ). The AUC for ATP/MV02 was 0.8381
(S.E. 0.1316) for the identification of NYHA-NR patients. No significant correlation was found between change in NYHA class and other metabolite fluxes/MVO2 or extraction fractions.

Identification of Complete Non-responders

There were 4 patients classified as C-NR (non-responders based on both LVEF and NYHA criteria), 15 patients classified as All-R (responders based on either LVEF or NYHA criteria or both), and 5 as C-R patients (responders based on both LVEF and NYHA criteria). C-NR patients demonstrated a greater mean FFA-ExtFx $(39 \% \pm 12 \%)$ than $\mathrm{C}-\mathrm{R}$ patients (mean FFA-ExtFx $=14 \% \pm 9 \%, p=0.0086$ ), as shown on box plots in Fig. 1 and Table 4. Metabolite fluxes/MVO2 for all metabolites demonstrating significant myocardial uptake or secretion in responders and non-responders to CRT are also compared in Table 4. CNR patients demonstrated a greater mean FFA/MVO2 $(0.115 \pm 0.112)$ than All-R patients (mean FFA $/ 02=0.034 \pm$ 0.030, $p=0.0171$ ), as shown in Fig. 1 and Table 4. The AUC for FFA/MVO2 was 0.8593 (S.E. 0.0965) and 0.8141 (S.E. 0.1159) for FFA-ExtFx in the identification of C-NR patients.

\section{Discussion}

In our study, $21 \%$ of patients with NIDCM who underwent CRT neither improved their NYHA class nor their LVEF during the mean follow-up period of more than 6 months.

Table 4 Metabolite extraction fractions (ExtFx) and metabolite fluxes corrected for MVO2 in non-responders and responders (mean \pm SD)

\begin{tabular}{|c|c|c|c|c|c|}
\hline & \multicolumn{2}{|l|}{ Non-Responders } & \multicolumn{3}{|l|}{ Responders } \\
\hline & LVEF-NR & C-NR & LVEF-R & C-R & All-R \\
\hline Alanine-ExtFx (\%) & $-9 \pm 10$ & $-13 \pm 13$ & $-8 \pm 13$ & $-15 \pm 13$ & $-7 \pm 11$ \\
\hline Aspartate-ExtFx (\%) & $-1 \pm 97$ & $-48 \pm 136$ & $7 \pm 21$ & $-2 \pm 4$ & $17 \pm 28$ \\
\hline FFA-ExtFx (\%) & $35 \pm 17 *$ & $39 \pm 12 * *$ & $18 \pm 10^{*}$ & $14 \pm 9 * *$ & $22 \pm 16$ \\
\hline Glucose-ExtFx (\%) & $4 \pm 7$ & $5 \pm 4$ & $4 \pm 19$ & $5 \pm 5$ & $4 \pm 17$ \\
\hline Glutamate-ExtFx (\%) & $41 \pm 27$ & $27 \pm 36$ & $32 \pm 20$ & $22 \pm 16$ & $40 \pm 20$ \\
\hline Lactate-ExtFx (\%) & $21 \pm 19$ & $5 \pm 10$ & $18 \pm 18$ & $4 \pm 10$ & $24 \pm 18$ \\
\hline O2-ExtFx (\%) & $58 \pm 18$ & $49 \pm 24$ & $57 \pm 12$ & $50 \pm 12$ & $59 \pm 12$ \\
\hline ATP/MVO2 & $4.72 \pm 0.34$ & $4.93 \pm 0.15$ & $4.94 \pm 0.11$ & $4.90 \pm 0.08$ & $4.78 \pm 0.31$ \\
\hline Alanine/MVO2 & $-0.006 \pm 0.008$ & $-0.010 \pm 0.010$ & $-0.005 \pm 0.008$ & $-0.008 \pm 0.006$ & $-0.004 \pm 0.007$ \\
\hline Aspartate/MVO2 & $0.001 \pm 0.002$ & $0.000 \pm 0.002$ & $0.000 \pm 0.000$ & $0.000 \pm 0.000$ & $0.000 \pm 0.001$ \\
\hline FFA/MVO2 & $0.077 \pm 0.084$ & $0.115 \pm 0.112 * * *$ & $0.027 \pm 0.017$ & $0.033 \pm 0.022$ & $0.034 \pm 0.030^{* * *}$ \\
\hline Glucose/MVO2 & $0.050 \pm 0.079$ & $0.055 \pm 0.027$ & $0.040 \pm 0.312$ & $0.069 \pm 0.068$ & $0.040 \pm 0.270$ \\
\hline Glutamate/MVO2 & $0.012 \pm 0.015$ & $0.014 \pm 0.023$ & $0.007 \pm 0.005$ & $0.007 \pm 0.007$ & $0.008 \pm 0.005$ \\
\hline Lactate/MVO2 & $0.063 \pm 0.069$ & $0.014 \pm 0.022$ & $0.049 \pm 0.065$ & $0.006 \pm 0.020$ & $0.068 \pm 0.071$ \\
\hline
\end{tabular}

Statistically significant differences between means $(p<0.05)$ are marked with $*$, **, and *** 

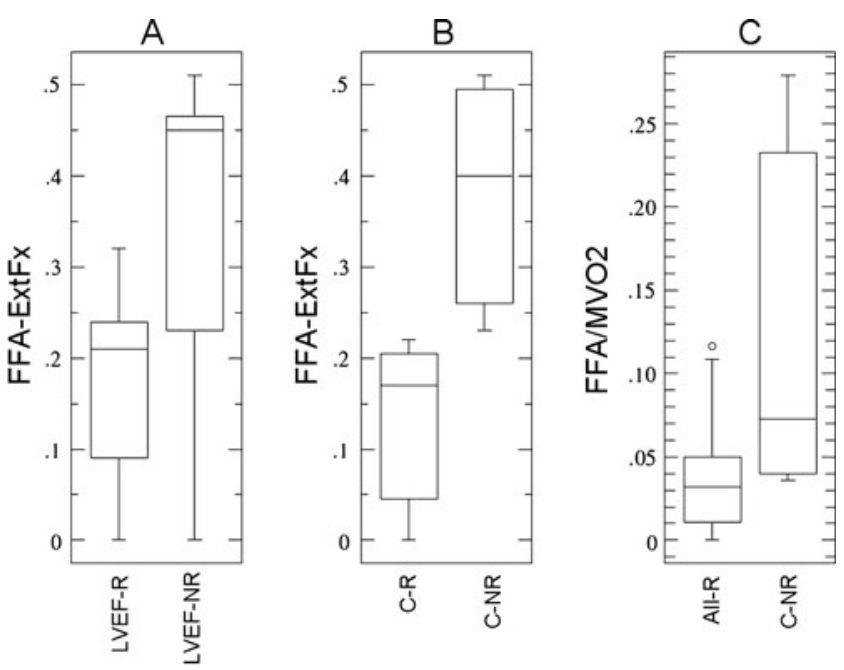

Fig. 1 Box plots comparing mean free fatty acid extraction fraction (FFA-ExtFx) for responders based on LVEF (LVEF-R) and nonresponders (LVEF-NR; a, $p=0.0098$ ) as well as complete responders $(\mathrm{C}-\mathrm{R})$ and complete non-responders (C-NR; $\mathbf{b}, p=0.0086)$. Box plots comparing mean FFA/MVO2 for all responders (All-R) and complete non-responders (C-NR; $\mathbf{c}, p=0.0171)$

These patients demonstrated greater myocardial uptake of FFA at the time of implantation than the $79 \%$ of patients who responded to CRT (Box plot c, Fig. 1). These findings suggested that myocardial FFA uptake flux may be a useful biomarker for identifying complete non-responders among non-ischemic heart failure patients undergoing CRT. The possibility of finding such a biomarker for complete nonresponders is intriguing, particularly, since we did not observe a significant correlation between the change in LVEF and change in NYHA class in our patient population. Therefore, ROC curves were constructed and the areas under the ROC curves were estimated to be 0.8593 for FFA/MVO2 data and 0.8141 for FFA-ExtFx in the identification of C-NR patients. If we were to use FFAExtFx as a biomarker for identifying C-NR patients, we could potentially avoid the error of excluding complete responders (C-R) from undergoing treatment, since we there was no overlap in FFA-ExtFx between complete nonresponder and complete responder groups (Box plot b, Fig. 1). Our study further demonstrated that patients who did not responded to BiV ICD implantation based strictly on the LVEF criteria also exhibited markedly greater myocardial FFA uptake rate than LVEF responders (Box plot a, Fig. 1). If we were to use FFA-ExtFx as a biomarker to identify LVEF-NR, we would prefer to set the cutoff point to a high specificity, such as $95 \%$, and the corresponding sensitivity would be estimated at $70 \%$.

It has been established that myocardial metabolism is altered in patients with heart failure (HF). However, studies of alterations in myocardial energy metabolism that may occur in heart failure have yielded conflicting results.
Several studies, including a study by Taylor et al., have demonstrated increased myocardial FFA metabolism and decreased myocardial glucose metabolism in patients with HF [10-12]. On the other hand, some animal studies and human studies, including a study by Dávila-Román et al., have suggested that substrate metabolism may revert to a fetal-like state with reduced FFA utilization and enhanced glucose uptake in the failing myocyte [13-16]. DávilaRomán et al. [16] compared myocardial FFA utilization (MFAU) and MVO2 in patients with Idiopathic Dilated Cardiomyopathy (IDCM) and normal controls. Mean MFAU in IDCM subjects $(134 \pm 44 \mathrm{nmol} / \mathrm{g} / \mathrm{min})$ was lower than MFAU in normal controls $(213 \pm 49 \mathrm{nmol} / \mathrm{g} / \mathrm{min})$. Mean MVO2 in IDCM patients $(4,935 \pm 770 \mathrm{nmol} / \mathrm{g} / \mathrm{min})$ was unchanged when compared with mean $\mathrm{MVO} 2$ in normal controls $(4,934 \pm 830 \mathrm{nmol} / \mathrm{g} / \mathrm{min})$. Therefore the MFAU/MVO2 in normal controls could be approximated as $213 / 4,934=0.0432$ and in IDCM as $134 / 4,935=0.0272$ for the Dávila-Román et al. study. In our study, All-R patients demonstrated mean FFA/MVO2 $(0.0343 \pm 0.0300)$ similar to the mean MFAU/MVO2 (of approximately 0.0272) reported in patients with IDCM in the study by DávilaRomán et al. [16]. C-NR patients in our study, on the other hand, demonstrated a hyper-elevated mean FFA/MVO2 $(0.1150 \pm 0.1118)$ that was greater than mean MFAU/MVO2 reported in IDCM patients (approximately 0.0272) by Dávila-Román et al. [16, 27], as well as in healthy older males $(0.035 \pm 0.001)$ and healthy younger males $(0.051 \pm$ 0.002). Therefore, the markedly increased myocardial FFA flux in our C-NR patients may be more closely related to findings reported in the study by Taylor et al. [11] who reported increased myocardial FFA metabolism in patients with HF. These authors concluded that myocardial fatty acid uptake rates in heart failure are higher than expected for the normal heart and that this shift of myocardial substrate use may be an indication of impaired energy efficiency in the failing heart, which could also be the case for our complete non-responder patient group [11].

It has been reported that myocardial contractile performance at a given MVO2 is lower when the heart is oxidizing fatty acids than when the heart is oxidizing other substrates such as glucose and lactate [9, 18-21]. A decrease in cardiac efficiency has been observed in healthy humans and experimental animals with elevated FFA during ischemia $[19,28-30]$. Increasing myocardial fatty acid uptake in dogs with an infusion of triglycerides resulted in an increase in MVO2 without improvement in mechanical power $[21,31]$. Furthermore, treatment with a partial fatty acid oxidation inhibitor ranolazine improved mechanical efficiency as well as left ventricular function in dogs with heart failure [32, 33]. In our study, complete non-responders demonstrated excessively elevated myocardial FFA uptake, which may have impaired mechanical 
efficiency of the myocardium with high fatty acid oxidation, resulting in a poor response to CRT.

In the FBA modeling platform, the possible ranges of all metabolic fluxes are first constrained by the stoichiometry of the reactions in the network due to conservation of mass balance and thermodynamics. Subsequently, additional constraints of measured metabolic fluxes are applied and a selected objective function flux that is of interest to the investigator can be calculated using linear programming. However, the application of too few measured fluxes as constraints will result in an unbounded solution space for the objective function flux. The abundance of myocardial metabolic data collected for NIDCM patients in this study allowed us to perform an analysis of the human cardiac mitochondria metabolic network using FBA. We hypothesized that failing myocytes would strive to maximize ATP production based on available substrates, and therefore, an objective function maximizing the ATP synthesis flux relative to available oxygen was chosen for the analysis of the human cardiac mitochondria metabolic network. In our study, patients with high ATP/MVO2 values were less likely to respond to CRT based on NYHA criteria. These findings may indicate that NYHA-NR patients may need to generate a greater amount of ATP relative to available oxygen to maintain basal cardiac workload, which may represent an already fully compensated metabolic state with no potential for improvement with resynchronization therapy [34].

One of the limitations of this study is that myocardial metabolite fluxes could only be expressed as values corrected for MVO2, or approximated by substrate extraction fractions, since myocardial blood flow measurements were not performed. Nevertheless, comparison of myocardial fluxes corrected for MVO2 may be more clinically relevant than evaluation of absolute fluxes. According to Kates et al. [35], correction of substrate fluxes with MVO2 may help to normalize the flux data for differences in workload between subjects of different ages, since the systolic and diastolic blood pressures are typically higher in the older subjects compared with younger subjects. Another limitation of the study concerns the fact that only NIDCM patients were enrolled in the protocol and the sample size was small. Although the patient population enrolled in the study was homogenous, it is not known if the results of this study can be generalized to all heart failure patients undergoing CRT. Nevertheless, we wished to avoid the confounding effects that $\mathrm{CAD}$ and ischemic cardiomyopathy can have on the segmental and global measurements of myocardial uptake of glucose, FFA, lactate, and other metabolites [36]. Finally, since this study did not involve radiolabeling of FFA, we were unable to differentiate between the handling of aqueous soluble and of lipid FFA fractions by myocardial tissue [37].
The results of our study indicate that myocardial FFA uptake flux might serve as a useful biomarker for identifying non-responders among NIDCM patients undergoing BiV ICD implantation. However, the process of data collection to obtain FFA-ExtFx is invasive, and the data becomes available only after the decision to implant BiV ICD device has been made. Therefore, a noninvasive test would be desirable, which could be carried out prior to the decision process of device implantation. Single photon emission computed tomography (SPECT) with 123I-15-( $\rho$-idophenyl)-3-methyl-pentadecanoic acid (BMIPP) offers the possibility of noninvasive semiquantitative assessment of regional FFA myocardial metabolism, which can be carried out on conventional nuclear medicine cameras that are widely available at diagnostic imaging facilities. Alternatively, positron emission tomography (PET) with C-11 palmitate could be utilized to assess regional myocardial metabolism quantitatively. Therefore, based on our invasive measurements of myocardial metabolism, we believe that a noninvasive prospective SPECT or PET imaging study is warranted, enrolling a large number of heart failure patients who are candidates for CRT.

Our results indicate that myocardial FFA flux may be helpful as a biomarker in identifying non-responders in NIDCM patients undergoing CRT. These findings further suggest that noninvasive imaging of myocardial FFA uptake in NIDCM patients prior to CRT with technologies, such as PET or SPECT, may be helpful in the selection process of potential non-responders and should be investigated prospectively in a large patient population. Furthermore, analysis of the myocardial metabolic network using a systems biology approach, such as FBA, may provide unique and clinically useful prognostic information in patients with NIDCM undergoing therapy.

Acknowledgements This work was supported by the UCSD Academic Senate Grant.

Conflicts of Interests None

Open Access This article is distributed under the terms of the Creative Commons Attribution Noncommercial License which permits any noncommercial use, distribution, and reproduction in any medium, provided the original author(s) and source are credited.

\section{References}

1. Cleland, J. G., Daubert, J. C., Erdmann, E., Freemantle, N., Gras, D., Kappenberger, L., et al. (2005). Cardiac resynchronizationheart failure (CARE-HF) study investigators. The effect of cardiac resynchronization on morbidity and mortality in heart failure. The New England Journal of Medicine, 352, 1539-1549 [PubMed: 15753115].

2. Bristow, M. R., Saxon, L. A., Boehmer, J., Krueger, S., Kass, D. A., De Marco, T., et al. (2004). Comparison of medical therapy, 
pacing, and defibrillation in heart failure (COMPANION) investigators. Cardiac-resynchronization therapy with or without an implantable defibrillator in advanced chronic heart failure. The New England Journal of Medicine, 350, 2140-2150 [PubMed: 15152059].

3. St John Sutton, M. G., Plappert, T., Abraham, W. T., Smith, A. L., DeLurgio, D. B., Leon, A. R., et al. (2003). Multicenter insync randomized clinical evaluation (MIRACLE) study group. Effect of cardiac resynchronization therapy on left ventricular size and function in chronic heart failure. Circulation, 107, 1985-1990 [PubMed: 12668512].

4. Abraham, W. T., Young, J. B., León, A. R., Adler, S., Bank, A. J., Hall, S. A., et al. (2004). Multicenter InSync ICD II Study Group. Effects of cardiac resynchronization on disease progression in patients with left ventricular systolic dysfunction, an indication for an implantable cardioverter-defibrillator, and mildly symptomatic chronic heart failure. Circulation, 110, 2864-2868 [PubMed: 15505095].

5. Saxon, L. A., \& Ellenbogen, K. A. (2003). Resynchronization therapy for the treatment of heart failure. Circulation, 108, 1044 1048 [PubMed: 12952826].

6. Beshai, J. F., Grimm, R. A., Nagueh, S. F., Baker, J. H., 2, Beau, S. L., Greenberg, S. M., et al. (2007). RethinQ Study Investigators. Cardiac-resynchronization therapy in heart failure with narrow QRS complexes. The New England Journal of Medicine, 357, 2461-2471 [PubMed: 17986493].

7. Chung, E. S., Leon, A. R., Tavazzi, L., Sun, J. P., Nihoyannopoulos, P., Merlino, J., et al. (2008). Results of the Predictors of Response to CRT (PROSPECT) trial. Circulation, 117, 2608-2616 [PubMed: 18458170].

8. Abraham, T., Kass, D., Tonti, G., Tomassoni, G. F., Abraham, W. T., Bax, J. J., et al. (2009). Imaging cardiac resynchronization therapy. JACC Cardiovascular Imaging, 2, 486-497 [PubMed: 19580733].

9. Stanley, W. C., Recchia, F. A., \& Lopaschuk, G. D. (2005). Myocardial substrate metabolism in the normal and failing heart. Physiological Reviews, 85, 1093-1129 [PubMed: 15987803].

10. Lommi, J., Kupari, M., \& Yki-Järvinen, H. (1998). Free fatty acid kinetics and oxidation in congestive heart failure. The American Journal of Cardiology, 81, 45-50 [PubMed: 9462605].

11. Taylor, M., Wallhaus, T. R., Degrado, T. R., Russell, D. C., Stanko, P., Nickles, R. J., et al. (2001). An evaluation of myocardial fatty acid and glucose uptake using PET with [18F] fluoro-6-thia-heptadecanoic acid and [18F]FDG in Patients with Congestive Heart Failure. Journal of Nuclear Medicine, 42, 55-62 [PubMed: 11197981].

12. Paolisso, G., Gambardella, A., Galzerano, D., D'Amore, A., Rubino, P., Verza, M., et al. (1994). Total-body and myocardial substrate oxidation in congestive heart failure. Metabolism, 43, 174-179 [PubMed: 8121298].

13. Rosenblatt-Velin, N., Montessuit, C., Papageorgiou, I., Terrand, J., \& Lerch, R. (2001). Postinfarction heart failure in rats is associated with upregulation of GLUT-1 and downregulation of genes of fatty acid metabolism. Cardiovascular Research, 52, 407-416 [PubMed: 11738057].

14. Barger, P. M., \& Kelly, D. P. (1999). Fatty acid utilization in the hypertrophied and failing heart: molecular regulatory mechanisms. The American Journal of the Medical Sciences, 318, 36-42 [PubMed: 10408759].

15. Sack, M. N., Rader, T. A., Park, S., Bastin, J., McCune, S. A., \& Kelly, D. P. (1996). Fatty acid oxidation enzyme gene expression is downregulated in the failing heart. Circulation, 94, 2837-2842 [PubMed: 8941110].

16. Dávila-Román, V. G., Vedala, G., Herrero, P., de las Fuentes, L., Rogers, J. G., Kelly, D. P., et al. (2002). Altered myocardial fatty acid and glucose metabolism in idiopathic dilated cardiomyopa- thy. Journal of the American College of Cardiology, 40, 271-277 [PubMed: 12106931].

17. Ramakrishna, R., Edwards, J. S., McCulloch, A., \& Palsson, B. O. (2001). Flux-balance analysis of mitochondrial energy metabolism: consequences of systemic stoichiometric constraints. American Journal of Physiology: Regulatory, Integrative and Comparative Physiology, 280, R695-R704 [PubMed: 11171647].

18. Burkhoff, D., Weiss, R. G., Schulman, S. P., Kalil-Filho, R., Wannenburg, T., \& Gerstenblith, G. (1991). Influence of metabolic substrate on rat heart function and metabolism at different coronary flows. The American Journal of Physiology, 261, H741H750 [PubMed: 1887921].

19. Korvald, C., Elvenes, O. P., \& Myrmel, T. (2000). Myocardial substrate metabolism influences left ventricular energetics in vivo. American Journal of Physiology. Heart and Circulatory Physiology, 278, H1345-H1351 [PubMed: 10749732].

20. Lammerant, J., Huynh-Thu, T., \& Kolanowski, J. (1985). Inhibitory effects of the $\mathrm{D}(-)$ isomer of 3-hydroxybutyrate on cardiac non-esterified fatty acid uptake and oxygen demand induced by norepinephrine in the intact dog. Journal of Molecular and Cellular Cardiology, 17, 421-433 [PubMed: 3894678].

21. Mjos, O. D. (1971). Effect of free fatty acids on myocardial function and oxygen consumption in intact dogs. The Journal of Clinical Investigation, 50, 1386-1389 [PubMed: 5090055].

22. Vanky, F. B., Hakanson, E., Szabó, Z., Jorfeldt, L., \& Svedjeholm, R. (2006). Myocardial metabolism before and after valve replacement for aortic stenosis. Journal of Cardiovascular Surgery (Torino), 47, 305-313 [PubMed: 16760867].

23. Vo, T. D., Greenberg, H. J., \& Palsson, B. O. (2004). Reconstruction and functional characterization of the human mitochondrial metabolic network based on proteomic and biochemical data. The Journal of Biological Chemistry, 279, 39532-39540 [PubMed: 15205464].

24. Lee, D. Y., Yun, H., Park, S., \& Lee, S. Y. (2003). MetaFluxNet: the management of metabolic reaction information and quantitative metabolic flux analysis. Bioinformatics, 19, 2144-2146 [PubMed: 14594721].

25. Bridge, P. D., \& Sawilowsky, S. S. (1999). Increasing physicians' awareness of the impact of statistics on research outcomes: comparative power of the $t$ test and Wilcoxon Rank-Sum test in small samples applied research. Journal of Clinical Epidemiology, 52, 229-235 [PubMed: 10210240].

26. Metz, C. E., Herman, B. A., \& Shen, J. H. (1998). Maximum likelihood estimation of receiver operating characteristic (ROC) curves from continuously-distributed data. Statistics in Medicine, 17, 1033-1053 [PubMed: 9612889].

27. Kates, A. M., Herrero, P., Dence, C., Soto, P., Srinivasan, M., Delano, D. G., et al. (2003). Impact of aging on substrate metabolism by the human heart. Journal of the American College of Cardiology, 41, 293-299 [PubMed: 12535825].

28. Kjekshus, J. K., \& Mjos, O. D. (1972). Effect of free fatty acids on myocardial function and metabolism in the ischemic dog heart. The Journal of Clinical Investigation, 51, 1767-1776 [PubMed: 5032525].

29. Mjos, O. D., Kjekshus, J. K., \& Lekven, J. (1974). Importance of free fatty acids as a determinant of myocardial oxygen consumption and myocardial ischemic injury during norepinephrine infusion in dogs. The Journal of Clinical Investigation, 53, 1290-1299 [PubMed: 4825226].

30. Simonsen, S., \& Kjekshus, J. K. (1978). The effect of free fatty acids on myocardial oxygen consumption during atrial pacing and catecholamine infusion in man. Circulation, 58, 484-491 [PubMed: 679439].

31. Mjös, O. D., \& Kjekshus, J. (1971). Increased local metabolic rate by free fatty acids in the intact dog heart. Scandinavian Journal of 
Clinical and Laboratory Investigation, 28, 389-393 [PubMed: 5157041].

32. Sabbah, H. N., Chandler, M. P., Mishima, T., Suzuki, G., Chaudhry, P., Nass, O., et al. (2002). Ranolazine, a partial fatty acid oxidation (pFOX) inhibitor, improves left ventricular function in dogs with chronic heart failure. Journal of Cardiac Failure, 8, 416-422 [PubMed: 12528095].

33. Chandler, M. P., Stanley, W. C., Morita, H., Suzuki, G., Roth, B. A., Blackburn, B., et al. (2002). Short-term treatment with ranolazine improves mechanical efficiency in dogs with chronic heart failure. Circulation Research, 91, 278-280 [PubMed: 12193459].

34. Wallhaus, T. R., Taylor, M., DeGrado, T. R., Russell, D. C., Stanko, P., Nickles, R. J., et al. (2001). Myocardial free fatty acid and glucose use after carvedilol treatment in patients with congestive heart failure. Circulation, 103, 2441-2446 [PubMed: 11369683].

35. Kates, A. M., Herrero, P., Dence, C., Soto, P., Srinivasan, M., Delano, D. G., et al. (2003). Impact of aging on substrate metabolism by the human heart. Journal of the American College of Cardiology, 41, 293-299 [PubMed: 12535825].

36. Wisneski, J. A., Gertz, E. W., Neese, R. A., \& Mayr, M. (1987). Myocardial metabolism of free fatty acids. Studies with 14Clabeled substrates in humans. The Journal of Clinical Investigation, 79(2), 359-366 [PubMed: 3805273].

37. Nellis, S. H., Liedtke, A. J., \& Renstrom, B. (1992). Fatty acid kinetics in aerobic myocardium: characteristics of tracer carbon entry and washout and influence of metabolic demand. Journal of Nuclear Medicine, 33(10), 1864-1874 [PubMed: 1403160]. 